\title{
Fabrication and Characterization of Hybrid Photovoltaic Devices Based On N-Type GaAs and Polymer Composites
}

\author{
Alireza Salehi $^{1}$, Paria Naderi ${ }^{1}$, F. A. Boroumand ${ }^{1}$, A. Dunbar ${ }^{2}$ \\ ${ }^{1}$ Department of Electronics, Faculty of Electrical Engineering, K.N.Toosi University of Technology \\ PO Box: 16315:1355, Tehran, Iran \\ salehi@kntu.ac.ir, paria.naderi@ee.kntu.com, boroumand@eetd.kntu.ac.ir \\ ${ }^{2}$ Chemical and Biological Engineering, University of Sheffield \\ Mappin Street, Sheffield, UK \\ a.dunbar@ sheffield.ac.uk
}

\begin{abstract}
Hybrid organic/inorganic solar cells have been fabricated and studied. The aim being to take advantage of the beneficial properties of both organic and inorganic materials. In this work we studied hybrid n-GaAs/Polymer photovoltaic devices and the effects of adding either carbon nanotubes or graphene into the polymer layer (either poly (3,4-ethyldioxythiophene):poly (styrenesulfunate) (PEDOT:PSS) or polyaniline (PANI)) to enhance its conductivity. We also investigated the use of two different metals ( $\mathrm{Al}$ or $\mathrm{Ag}$ ) as the anode electrode material to determine the influence of metal atoms migrating into the polymer layer on solar cell efficiency. The devices with the structure Al/PEDOT:PSS:MWCNT/n-GaAs/Au:Ge/Ni/Au exhibited the best performance, with a short circuit current (Jsc) of $28.6 \mathrm{~mA} / \mathrm{cm} 2$, an open circuit voltage ( $\mathrm{Voc}$ ) of $0.51 \mathrm{~V}$, a fill factor (FF) of $29.8 \%$ and a power conversion efficiency (PCE) of $4.41 \%$ which is higher than other inorganic/organic hybrid devices reported in the literature. Based on optical absorption measurements and SEM analysis the solar cell performances reported here can mainly be attributed to photon absorption in both the doped PEDOT:PSS and the GaAs layers resulting in an increase of electron-hole pairs and improved the efficiency of the solar cells containing PEDOT:PSS in comparison with those containing PANI.
\end{abstract}

Keywords: Organic/Inorganic, Solar Cell, GaAs/Polymar, PEDOT:PSS, PANI.

\section{Introduction}

Organic/inorganic hybrid solar cells incorporating polymers have attracted the growing interest of researchers and industries due to their simple fabrication procedure and relatively high efficiency $[1,2]$. Hybrid solar cells are usually made of p-type polymers deposited on n-type inorganic semiconductors [3, 4]. One of the main advantages of using organic materials in photovoltaic devices is that they permit solution phase processing techniques such as inject printing or roll-to-roll techniques [5]. On the other hand different inorganic semiconductors such as crystalline Si or GaAs solar cells dominate the market of photovoltaic devices as these provide very high power conversion efficiency [5, 6]. However the processing techniques required to manufacture these devices are very expensive.

Hybrid organic/inorganic solar cells utilizing conducting polymers and inorganic semiconductors like GaAs should have potential to achieve high PCE but regrettably currently efficiencies are still very low. Ren et al. [7] reported photovoltaic devices using GaAs nanowires and conjugated polymer P3HT and obtained a PCE of 2.36\%. Liang Yan et al. [1] investigated hybrid solar cells using different polymers deposited on $\mathrm{n}-\mathrm{GaAs}$ and reported various PCEs ranging from very low $\sim 0.02 \%$ up to $2.75 \%$. Therefore, there is a demand to conduct further research to understand the factors that are limiting their performance and therefore unlock the possible advantages of using both organic and inorganic materials.

In a hybrid solar cell the incident light is primarily absorbed by the n-type inorganic semiconductor substrate and a hole transport layer (HTL), made from a thin organic layer is used to extract the holes generated in the inorganic substrate and selectively transport them into the metallic anode from where they flow into the external circuit [8].

For the organic part, conducting polymers are used, because of their optical and electrical properties and possible flexibility [9-12]. The conjugated polymer blend, PEDOT:PSS, is a good example of such an organic material [3]. PEDOT:PSS is attractive as a material for organic electronics due to its high conductivity $(0.1$ to $1 \times 103 \mathrm{~S} / \mathrm{cm})$ and high solubility in polar solvents [13]. Another conjugated polymer which is being investigated nowadays in solar cells, is PANI. 
PANI was discovered to have the characteristic of being able to switch between acting as a conductor and an insulator under certain experimental conditions [14]. Since then, PANI has become a subject of great interest in research [15]. PANI is a promising organic electronic material due to its unique conduction mechanism and its high environmental stability [16, 17]. The chemical structures of these polymers are shown in Figure 1. However, the moderate conductivity of these both polymers (PEDOT:PSS and PANI) is still a limiting factor in solar applications. But, by addition of certain materials the conductivity can be significantly increased which influences the characteristics of the resulting solar cell devices [18].

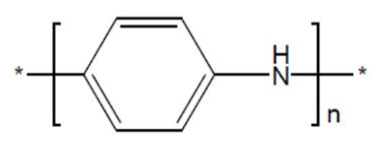

Polyaniline

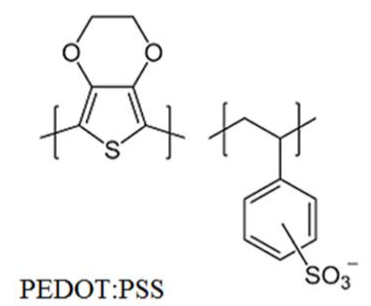

Fig. 1: Molecular structures of two conducting polymers: Polyaniline (PANI) and PEDOT:PSS.

Multiwall carbon nanotubes (MWCNTs) are an appropriate choice of additive, because of their high electrical and thermal conductivity. When using PEDOT:PSS as a HTL it is known that adding MWCNTs result in phase separation of the PEDOT and PSS which helps establish improved electrical interconnections between the separated PEDOT islands (conductive phase) which are dispersed within the insulating PSS phase [19]. The PEDOT backbone contains aromatic thiophene rings, which enable strong $\pi-\pi$ stacking interactions with the hybridized carbon on the surface of the MWCNTs. This interaction with PEDOT helps to separate the otherwise agglomerated MWCNTs and therefore improves their dispersion [20, 21]. PSS acts as a surfactant to disperse the MWCNTs; the long molecular chain of PSS wraps around the MWCNTs to separate them from each other [22, 23].

Graphene, in any of its 2D, 1D or 0D forms, also has great potential in nano-electronic applications [24, 25]. It has been reported that organic materials with high conductivity, transparency, and flexibility can be produced which result in reasonable solar cell characteristics, if graphene-based materials are used in an appropriate form. The additive acts as a doping material for the conducting polymers [26]. On the other hand, using graphene as an additive highly dispersed within PANI should increase carrier density within the organic layer therefore increasing the electroactive region of the polymer as well as the conductivity of the composite material [27].

We also investigated the two different anode metals ( $\mathrm{Al}$ or $\mathrm{Ag}$ ) to determine the influence of metal atoms migrating into the polymer on solar cell efficiency. $\mathrm{Al}$ and $\mathrm{Ag}$ were chosen because the work functions of $\mathrm{Al}(4.31 \mathrm{eV})$ and $\mathrm{Ag}(4.35) \mathrm{eV}$ ) are very close to each other [28], so any differences in characteristics are likely to be attributable to the migration of silver atoms penetrating the organic layer [29] and forming path ways for electron conduction into the organic layer.

Significant progress has been achieved in developing higher efficiency organic solar cells but their performances still lag behind those of inorganic solar cells. The role of different polymeric materials with various additives are still not fully understood. In this study, hybrid organic/inorganic solar cells produced using n-type GaAs as the inorganic substrate and doped conductive polymers PEDOT:PSS and PANI as the organic layers. Carbon nanostructures, including 1D carbon nanotubes (MWCNTs) and graphene nanoparticles (GNP) have been used as the dopants within the polymers. The results presented here show that an efficient solar cell can be fabricated from doped organic materials deposited on n-GaAs substrate with only a few simple processing steps exhibiting PCE above $4.4 \%$ which is much higher than that of organic materials without GaAs substrate.. 


\section{Experimental Section (Materials, Fabrication Techniques and Characterisation Methods)}

The inorganic substrates used in this study were $\mathrm{n} / \mathrm{n}+\mathrm{GaAs}$ wafers with carrier concentrations of $1 \times 10^{15} \mathrm{~cm}^{-3}$ and $5 \times 10^{18} \mathrm{~cm}^{-3}$, respectively. The wafer was cut into $7 \mathrm{~mm} \times 6 \mathrm{~mm}$ pieces and two cells were fabricated on each substrate. The cleaning process used included the following steps: $10 \mathrm{~min}$ ultra-sonication in each of acetone, deionised (DI) water, $\mathrm{HCl}$ and DI water again. Finally, the substrates were dried with nitrogen gas. In order to form Ohmic contacts for the back electrodes, a $100 \mathrm{~nm}$ layer of $\mathrm{Au}$ :Ge alloy was deposited by thermal vacuum deposition at a pressure of $10^{-5}$ Torr, followed by a Ni layer with an approximate thickness of 30nm, and finally $100 \mathrm{~nm}$ of Au was deposited on the back surface. Subsequently this was annealed at $350^{\circ} \mathrm{C}$ for 3 minutes in nitrogen gas to form a good Ohmic contact [30].

The PEDOT:PSS solution with a ratio of 1:2 wt\% in water was purchased from Sigma Aldrich. The Poly Aniline (PANI) was synthesised by polymerisation of aniline using ammonium persulfate in hydrochloric acid. The aniline monomer $(1 \mathrm{~mL})$ was dissolved in $20 \mathrm{~mL}$ aqueous hydrochloric acid $(1 \mathrm{M})$ and then DI water $(15.5 \mathrm{~mL})$ was added. Ammonium Persulfate (APS) oxidant (1.2 g) also was dissolved in $10 \mathrm{~mL}$ aqueous hydrochloric acid (1M) and stirred for $15 \mathrm{~min}$ at room temperature. The aqueous APS solution was then added to the aniline/ $\mathrm{HCl}$ mixture. Finally, after polymerisation had occurred the material exhibited a green colour which indicates the formation of PANI emeraldine salt. Subsequently different dopants of MWCNTs $(30-50 \mathrm{~nm}$ in diameter purchased from NanoSav) and graphene (nanoparticles of 40-70 nm in diameter purchased from NanoSav) were used as additives within the organic materials. They were dissolved separately in both PEDOT:PSS and PANI solutions resulting in blends with MWCNT and GNP concentrations of $6 \mathrm{mg} / \mathrm{ml}$; this was achieved by using $150 \mu \mathrm{l}$ of the diluted PEDOT:PSS or PANI with $1 \mathrm{mg}$ of the nanostructures. The prepared solutions were then ultra-sonicated for 1 hour to allow the particles to be dispersed homogeneously. Finally, the dispersion solutions were drop-cast onto the clean n-GaAs substrate. The samples were subsequently thermally annealed at $60^{\circ} \mathrm{C}$ for 30 minutes in ambient atmosphere in order to remove residual solvent from the organic layer.

Aluminium or silver was deposited through a $1 \mathrm{~mm} \times 1 \mathrm{~mm}$ metal mask defining the anode electrodes for the devices on each substrate; this was achieved in a vacuum evaporation system at a pressure of 10-5 Torr. A Keithley-238 High Current Source Measure Unit was then used to measure the J-V characteristics of the resulting solar cells both in dark and under illumination with a solar simulator producing AM1.5 illuminations with an intensity of $100 \mathrm{~mW} / \mathrm{cm} 2$. The absorption coefficient of the layers was measured using a Perkin Elmer spectroscope with a wavelength range from 350 to $1000 \mathrm{~nm}$ and the SEM micrographs were obtained using a MIRAN TESCANSEM at operating at $15 \mathrm{kV}$.

The architecture used for the complete devices is shown in Figure 2 and consists of a small area of Metal Anode (Ag or Al)/ Conducting Polymer (undoped or doped with MWCNTs or GNP)/n-GaAs/Metal Cathode (AuGe/Ni/Au). The J-V characteristics of the devices were studied both as diodes in the dark and as photovoltaic devices under illumination. A relationship between the illuminated (solar) and dark (diode) characteristics was noted, highlighting the fact that in order to have a high-performance solar cell, the properties of the diode junction play a significant role.

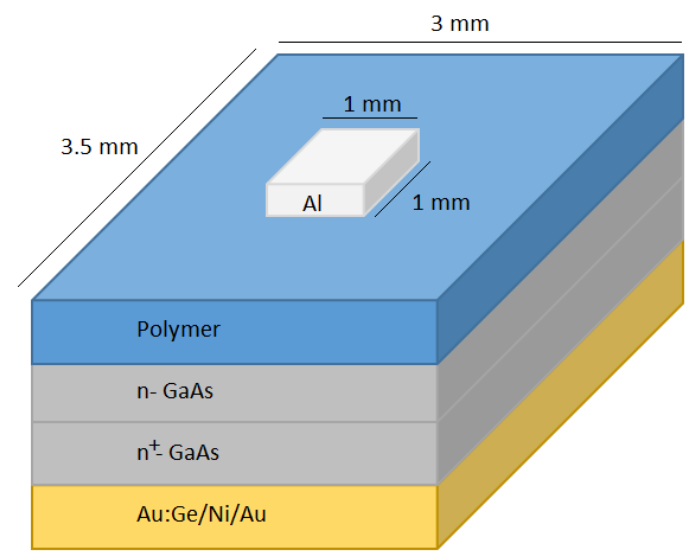

Fig. 2: Schematic picture of the devices fabricated in this study.

\section{Results and Discussions}


Two different conducting polymers with two different dopants deposited on the n-GaAs substrates resulting in four different cells for investigation. For each set of experiments more than ten devices were fabricated to ensure reliable characteristics. Slight differences were observed between the nominally identical devices. The numerical results reported here are of the representative average of the similar devices produced and the J-V curves are from the best performing device for each structure. The organic layers of the devices consisted of one of the following composites: PEDOT:PSS:MWCNT, PEDOT:PSS:GNP, PANI:MWCNT and PANI:GNP. Control devices were also made without any additives in the organic layers and it is worth noting that samples where the diode junction consisted of PEDOT:PSS/GaAs and PANI/GaAs revealed much lower PCE than the samples fabricated with additives in the organic layers. PEDOT:PSS/GaAs and PANI/GaAs revealed average PCEs of $0.15 \%$ and $0.064 \%$, respectively. Overall the devices with PEDOT:PSS composites showed the best performances. The PCEs for PEDOTT:PSS:MWCNT/n-GaAs and PEDOT:PSS:GNP/n-GaAs with Al electrodes have been measured as $4.41 \%$ and $4.11 \%$, respectively.

In order to study the influence of the metal atoms migrating into the polymer layer devices were made with the best performing composite PEDOT:PSS:MWCNT and two different anode metals. Some samples had Ag anodes, and some had $\mathrm{Al}$ anodes and they showed significantly different performances. The J-V characteristics of the devices with $\mathrm{Ag}$ anode revealed poor performance compared to that with $\mathrm{Al}$ anode shown in Figure 3.

The samples with a PEDOT:PSS:MWCNT layer fabricated with a Ag anode exhibited the following solar cell characteristics: $\mathrm{Vop}=0.46 \mathrm{~V}, \mathrm{Jsc}=20 \mathrm{~mA} / \mathrm{cm} 2, \mathrm{FF}=36 \%$, and a PCE of $3.3 \%$. These different characteristics are tentatively attributed to the migration of silver atoms into the organic layer [29] to form electron conduction pathways within the organic layer. The transport of electrons along these pathways provides a source of electrons to recombine with the holes being transported through the polymer layer. This results in a large number of recombination centres where detrimental electron-hole recombination occurs and subsequently reduces the device performance. The devices with Al electrodes exhibited higher PCEs, which may be because the Al does not migrate to the same extent.

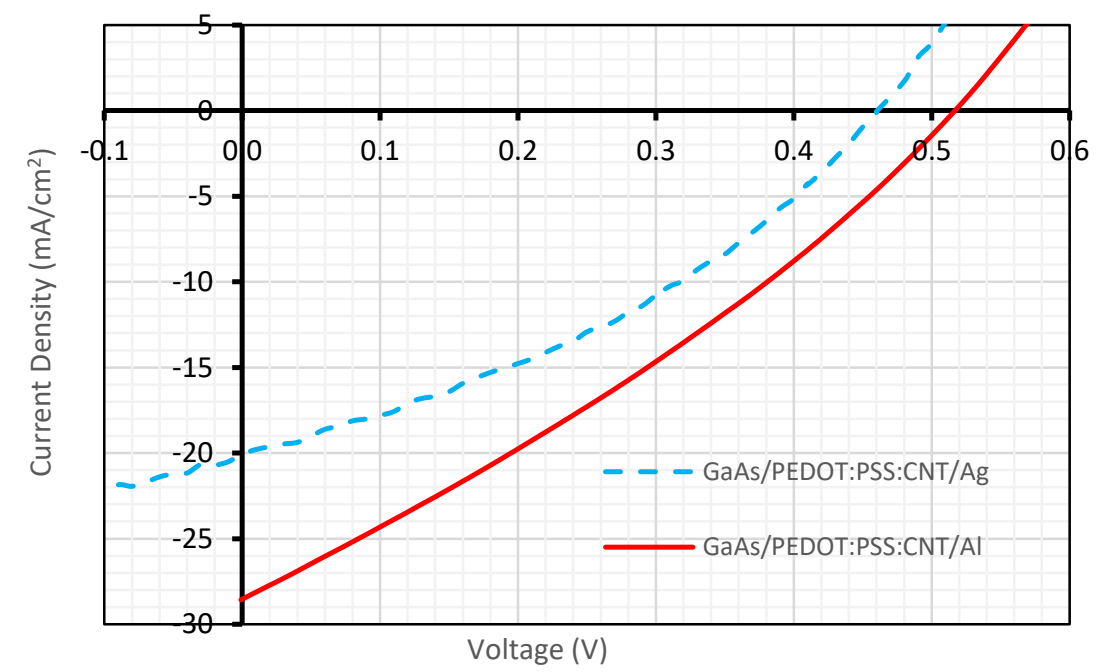

Fig. 3: Current voltage characteristics of two different samples: Ag/PEDOT:PSS:MWCNT/GaAs and Al/PEDOT:PSS:MWCNT/GaAs.

Figure 4 shows the J-V characteristics of the four devices doped with GNP and MWCNT and prepared with Al electrodes. It is seen that the samples with doped PEDOT:PSS organic layers deposited on GaAs exhibit significantly higher performance than the samples fabricated with doped PANI. The results of the solar cells fabricated here are summarized in Table 1. The devices with Al/PEDOT:PSS:MWCNT/n-GaAs have the best performance with a PCE of $4.41 \%$ when compared to the other devices considered here. Further, it is obvious that the Jsc and FF have been enhanced in the doped PEDOT:PSS/n-GaAs devices compared to the PANI/n-GaAs devices which have been doped 
with similar additives. It is noteworthy that our hybrid organic/GaAs solar cells with $\mathrm{Al}$ electrodes demonstrate better performances than similar devices reported in literature which have achieved up to 2.75\%. [1].

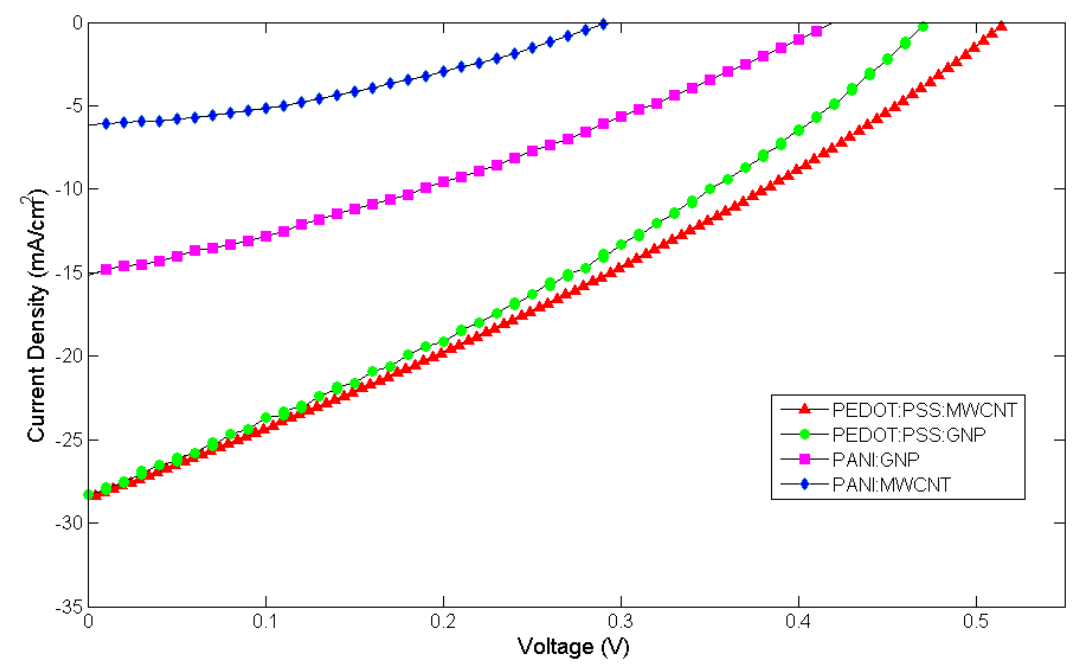

Fig. 4: J-V characteristics of the four solar cells with the conducting polymers PEDOT:PSS and PANI doped with GNP and MWCNT. $\mathrm{Al}$ was used for the anode electrode in these devices which were tested under $100 \mathrm{~mW} / \mathrm{cm} 2 \mathrm{AM} 1.5$ illumination.

Table 1: Solar cell characteristics of four different devices reported in this study.

\begin{tabular}{|c|l|l|l|l|}
\hline Organic Compound & VOP $(\mathrm{V})$ & $\mathrm{JSC}\left(\mathrm{mA} / \mathrm{cm}^{2}\right)$ & FF $(\%)$ & PCE (\%) \\
\hline PEDOT:PSS:MWCNT & 0.51 & 28.6 & 29.8 & 4.41 \\
\hline PEDOT:PSS:GNP & 0.47 & 28.4 & 30.4 & 4.11 \\
\hline PANI:GNP & 0.42 & 15.2 & 30.8 & 1.92 \\
\hline PANI:MWCNT & 0.3 & 6.12 & 34 & 0.63 \\
\hline
\end{tabular}

The doped PANI devices shown in Fig. 4 exhibit lower short circuit currents (Jsc) and lower open circuit voltages (Voc) than the doped PEDOT:PSS devices. To explain these differences, we can refer to the energy band diagrams of the devices studied here. As illustrated in Fig. 5 PEDOT:PSS has a HOMO level higher than the valence band of the GaAs semiconductor, whereas the HOMO levels for PANI have been reported above and below the GaAs valance band. Therefore, the PANI may present a small barrier to hole extraction from the PANI devices since the polymer HOMO levels play the role of the hole transporting layer which permits the holes to pass across the GaAs/polymer junction from the GaAs into the organic material. Meanwhile, the LUMO levels in both PEDOT:PSS and PANI are higher than the conduction band of the GaAs, and as a result these block electron transport across the interface and therefore minimizes any electron-hole recombination in the organic layer. Moreover, we have measured the absorption coefficient of the two organic layers deposited on glass substrates from 350 to $1000 \mathrm{~nm}$ wavelength. We have found that the absorbance of PEDOT:PSS:MWCNT was in the range of 0.6 A.U., whereas the absorption coefficient of PEDOT:PSS:GNP was measured in the range of 0.45 A.U. This indicates that the devices made with MWCNT as the additive absorbed more photons within the organic layer that the GNP doped devices. This helps to ensure that the electron hole pairs are generated close to the polymer/GaAs interface and therefore are more likely to be transported across the interface due to the differences in electronic energy level. This means that more of the generated electron-hole pairs will be separated and captured as useful charge which results in higher solar cell efficiency. 


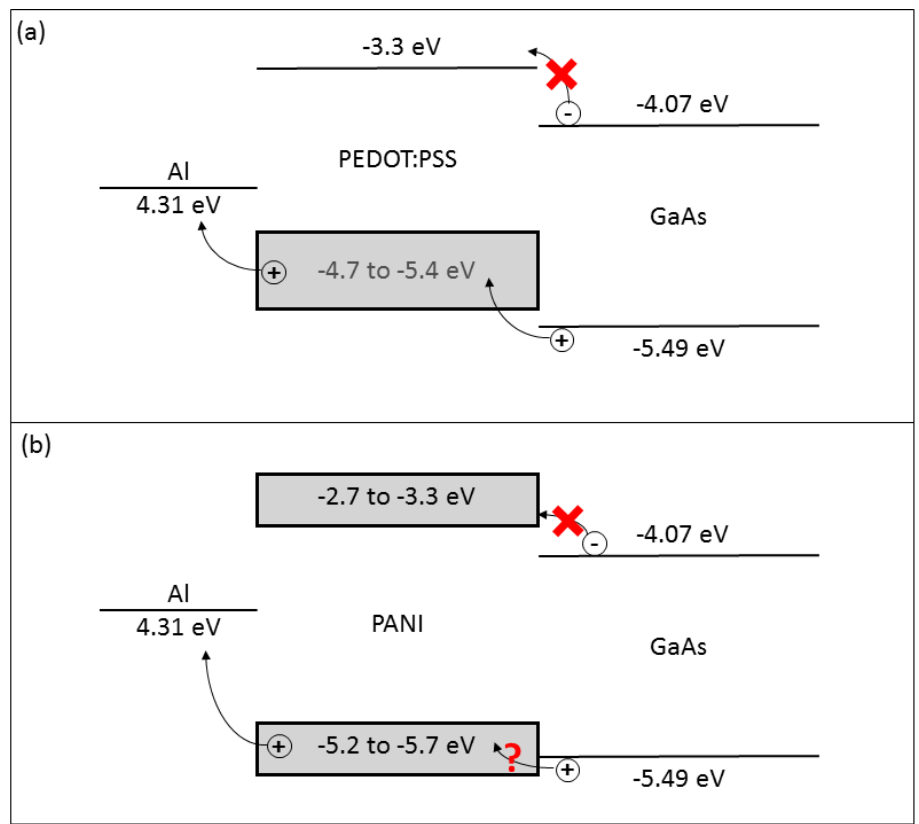

Fig. 5: Energy band diagrams of two hybrid solar cells with a)PEDOT:PSS [31, 32] and b)PANI [33, 34] as the hole transporting layers deposited on $n-G a A s$ substrates. It indicates the transport route for holes travelling from the GaAs through the polymers to the Al anode and that electron transport via this route is blocked.

SEM micrographs of the four polymer composites are shown in Figure 6. Comparing Figures 6(a) \& (b), and with Figures 6(c) and (d) it is obvious that the dispersion of the nanostructured dopants (GNP and MWCNT) is notably different for the PEDOT:PSS and PANI samples. It appears that in the PEDOT:PSS samples the corresponding MWCNT fibres and GNPs are more discrete. For the Figure 6(a) there are discrete fibres of aggregated MWCNTs and in Figure 6(b) discrete spherical particles of aggregated GNP. Both types of film result in an open porous structure which is presumably impregnated with a PEDOT:PSS coating. In contrast, the in the PANI samples the MWCNT fibres and GNPs are embedded within a more continuous amorphous matrix of PANI. Since the PEDOT:PSS appears to coat the MWCNTs and graphene whereas the PANI tends to encase them in a thin coating combined with the higher conductivity, $1 \mathrm{~S} / \mathrm{cm}$ (from supplier data), of undoped PEDOT:PSS it is expected that carriers will flow more easily through in the PEDOT:PSS layers compared to the PANI layers, where the PANI conductivity [35] is expected to be in the range $10^{-5}$ to $10^{-2} \mathrm{~S} / \mathrm{cm}$ and the high aspect ratio of the MWCNT fibres further increases the PEDOT:PSS:MWCNT film performance over the spherical GNPs.

(a)

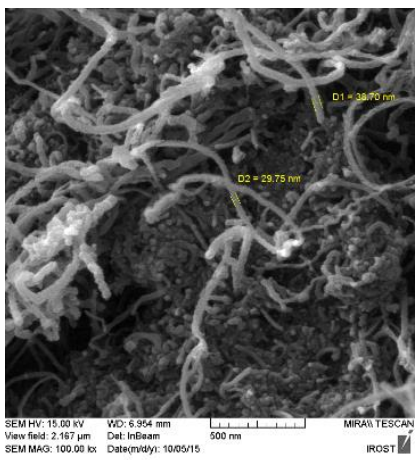

(b)

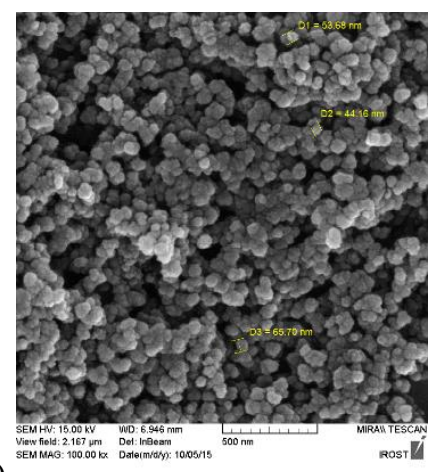

) 

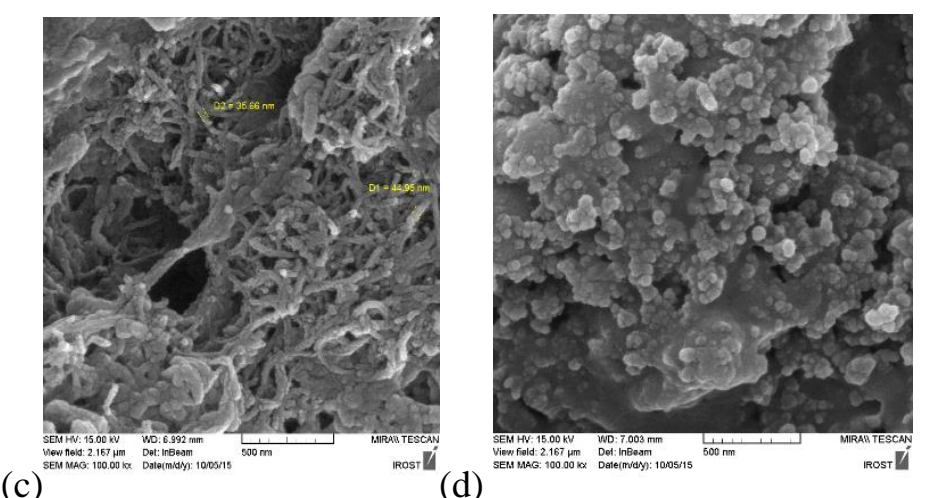

Fig. 6: SEM images of the surface of the four devices with layers (a) PEDOT:PSS:MWCNT, (b) PEDOT:PSS:GNP, (c)

PANI:MWCNT and (d) PANI:GNP.

In order to examine the properties of the junctions formed between the layers in the fabricated devices, the dark current voltage characteristics of the devices were studied. The J-V characteristics of the four types of device fabricated are shown on a linear scale in Figure 7(a) and Figure 7(b) shows the same data on a semi logarithmic scale.
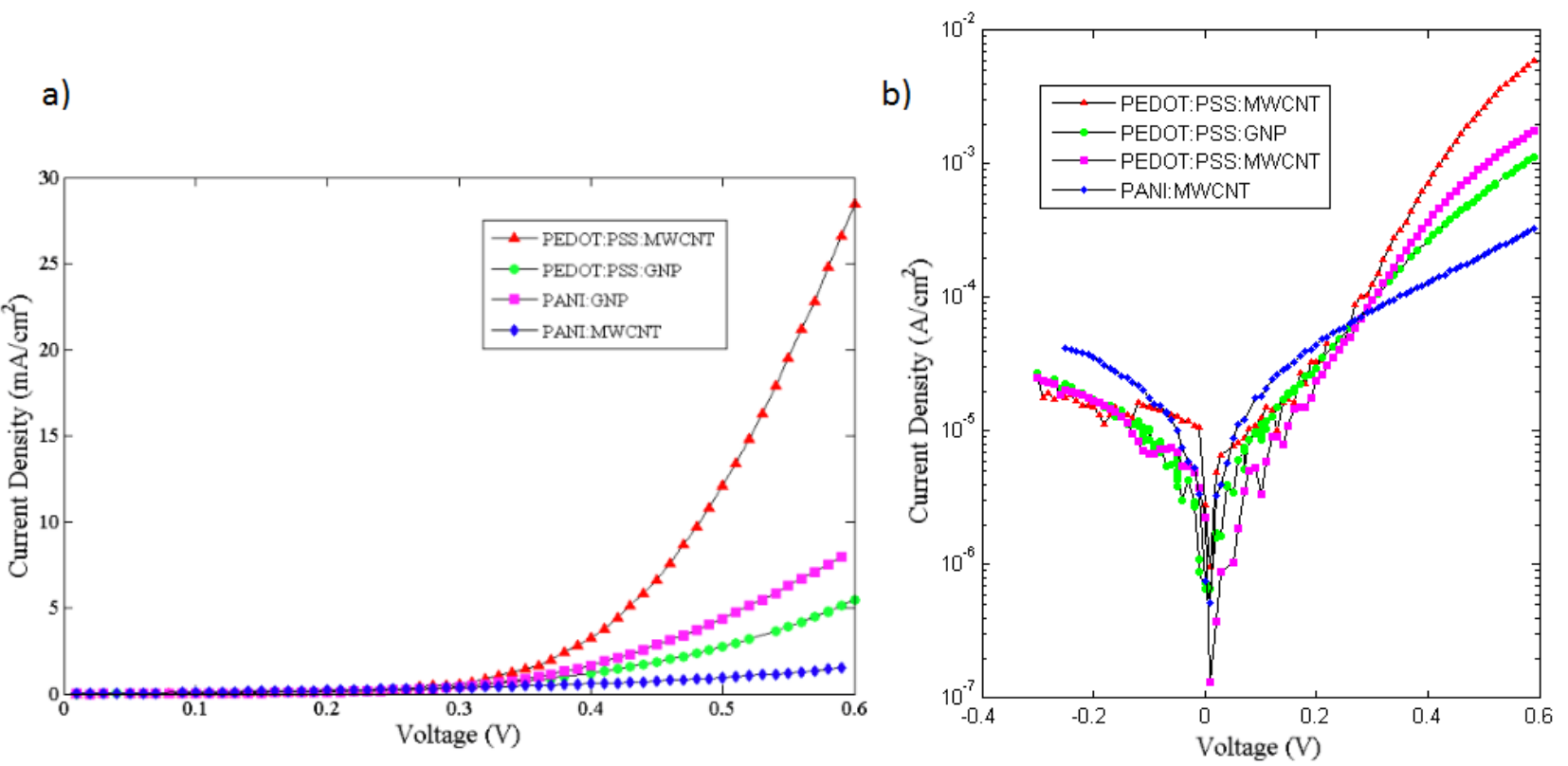

Fig. 7: (a) The Current Voltage (J-V) characteristics of the fabricated devices recorded in the dark and (b) the same data plotted on a semi-logarithmic scale.

To obtain the saturation current this was estimated using the intersect value of the extension of the linear part of J-V curves in Fig. 7(b) and the current axis at zero bias. The barrier height of the junction $\Phi_{\mathrm{B}}$ was calculated using following equation $[1,30]$ :

$$
\Phi_{\mathrm{B}}=\left(\frac{\mathrm{kT}}{q}\right) \ln \left(\frac{\ddot{\mathrm{A}} \mathrm{T}^{2}}{\mathrm{~J}_{\mathrm{s}}}\right)
$$

Where, Js is the saturation current density, $\mathrm{q}$ is the absolute electronic charge, $\ddot{A}$ is the effective Richardson constant which equals $4.4 \mathrm{~A} \mathrm{~cm}-2 \mathrm{~K}-2$ for n-type GaAs, $\mathrm{k}$ is the Boltzmann constant, and $\mathrm{T}$ is the absolute temperature. The values of saturated currents and junction barrier heights are summarized in Table 2. 
Table 2: The saturation current densities and the barrier heights of the junctions of the devices measured in dark.

\begin{tabular}{|l|l|l|}
\hline Organic Compound & Js $\left(\mathrm{A} / \mathrm{cm}^{2}\right)$ & Barrier Height $(\mathrm{eV})$ \\
\hline PEDOT:PSS:MWCNT & $8 \times 10^{-6}$ & 0.664 \\
\hline PEDOT:PSS:GNP & $1.6 \times 10^{-5}$ & 0.658 \\
\hline PANI:GNP & $6 \times 10^{-6}$ & 0.671 \\
\hline PANI:MWCNT & $6.5 \times 10^{-5}$ & 0.608 \\
\hline
\end{tabular}

We have found that there is a relationship between the barrier height of the junctions formed between the layers of the devices and their solar characteristics. Comparing the parameters summarized in Tables 1 and 2, it is evident that for each specific organic layer a higher barrier height results in a better performance of the solar cell. Presumably a large barrier height is helpful in reducing leakage currents detrimental to performance. However, it is noted that the differences in barrier height are fairly small so this is not thought to be the dominant parameter that determine the device performance.

In order to investigate the current transport between different layers of the devices, the diode ideality factor, $\mathrm{n}$, and the series resistance, Rs, were calculated. The results are summarized in Table 3. This was calculated using the J-V curves shown in fig. 7(b) with the equation:

$$
\frac{\mathrm{dV}}{\mathrm{d}(\ln (\mathrm{J}))}=\mathrm{JR}_{\mathrm{s}}+\mathrm{n}\left(\frac{\mathrm{kT}}{\mathrm{q}}\right)
$$

Where Rs is the series resistance of the device which dominates in the high bias region as observed in Fig. 7(b), $n$ is the ideality factor and is a measure of diode conformity to pure thermionic emission [30].

Table 3: Ideality factor and series resistance of the fabricated devices.

\begin{tabular}{|l|l|l|}
\hline Organic Compound & $\mathrm{n}$ & Rs $(\Omega)$ \\
\hline PEDOT:PSS:MWCNT & 2.05 & 345 \\
\hline PEDOT:PSS:GNP & 3.9 & 825 \\
\hline PANI:GNP & 2.39 & 1494 \\
\hline PANI:MWCNT & 5.58 & 3659 \\
\hline
\end{tabular}

Considering the values in Tables 1 and 3 , it is clear that better junction quality i.e. decreased ideality factor appears to result in better cell performance. Comparing the results given above it is obvious that of those tested samples the solar cell structure of Al/PEDOT:PSS:MWCNT/n-GaAs shows the highest efficiency as a solar cell and the lowest ideality factor as well as the lowest series resistance as a diode. Furthermore, comparing the solar cells characteristics of the devices using PEDOT:PSS and PANI on GaAs shown in Fig. 4 and Table 1 and taking into account the very high series resistances (Rs) of PANI devices it is obvious that these devices whether doped with GNP or MWCNT show significantly lower solar cell performance. This is likely due to poorer conductivity through the PANI composite layer and therefore reduced charge carrier injection to the electrodes representing increased series resistance [5]. Jian- Jhou Zeng et al. [3] reported hybrid photovoltaic devices of reduced carbon oxide (RCO)-based PEDOT:PSS/n-GaAs and have found that RCO doping might lead to a reduction in the series resistance of their devices which is consistent with our results. The series resistance is thought to be the dominant parameter limiting the device performance and therefore future research will aim to further reduce this with a view to improving device performance.

\section{Conclusion}

In summary, hybrid organic/inorganic solar cells using n-GaAs as the light-absorbing substrate and the conducting polymers PEDOT:PSS and PANI as the hole transporting layers were fabricated and investigated. Two different additives were used to dope the polymer layers. Using MWCNTs as an additive in PEDOT:PSS deposited on GaAs substrate improved the device performance significantly. The maximum PCE of $4.41 \%$ was achieved for the devices with the structure Al/PEDOT:PSS:MWCNT/n-GaAs/Au:Ge/Ni/Au, which is much higher than the devices 
fabricated by PANI on GaAs substrate. Doping the PEDOT:PSS with MWCNTs as an additive significantly improves the device performance. It is shown to modify the doped polymer film structure, increase its conductivity and increase the optical absorption within this layer also. Therefore the observed enhancement in device PCE is attributed to the higher conductivity of the PEDOT:PSS layer and an in the improvement of photon absorption within the device. This absorption occurs within both the doped organic layers and within the direct band gap inorganic semiconductor substrate.

Finally, we can conclude that by adding MWCNTs and to a lesser extent GNP to the polymer layer within hybrid organic/inorganic solar cells it is possible to improve their performance. The devices presented here provide a guideline for further developments towards the optimization of hybrid solar cell performance.

Compliance with Ethical Standards: The authors declare that they have no conflict of interest.

\section{References}

[1] L. Yan, W. You, "Real Function of Semiconducting Polymer in GaAs/Polymer Planar Heterojunction Solar Cells," ACS Nano, vol. 7, pp. 6619-6626, 2013.

[2] K. Y. Cheng, H. T. Pan, S. C. Yu, W. S. Weng, Y. C. Lai, Y. C. Lin, Y. C. Chen, M. C. Li, H. W. Hu, P. Yu, H. F. Meng, "10\% efficiency hybrid GaAs/PEDOT:PSS solar cells with monolayer graphene," in 2014 IEEE 40th Photovoltaic Specialist Conference (PVSC), pp. 1519-1521, 2014.

[3] J.-J. Zeng, C.-L. Tsai, Y.-J. Lin, "Hybrid photovoltaic devices based on the reduced graphene oxide-based polymer composite and n-type GaAs," Synthetic Metals, vol. 162, pp. 1411-1415, 2012.

[4] C. Jiun-Jie, S. Shu-Chia, H. Shih-Che, L. Ching-Fuh, "GaAs nanowire/poly $(3,4$ ethylenedioxythiophene):poly(styrenesulfonate) hybrid solar cells," Nanotechnology, vol. 21, p. 285203, 2010.

[5] M. Wright, A. Uddin, "Organic — inorganic hybrid solar cells: A comparative review," Solar Energy Materials and Solar Cells, vol. 107, pp. 87-111, 2012.

[6] G. J. Bauhuis, P. Mulder, E. J. Haverkamp, J. C. C. M. Huijben, J. J. Schermer, " $26.1 \%$ thin-film GaAs solar cell using epitaxial lift-off," Solar Energy Materials and Solar Cells, vol. 93, pp. 1488-1491, 2009.

[7] S. Ren, N. Zhao, S. C. Crawford, M. Tambe, V. Bulović, S. Gradečak, "Heterojunction Photovoltaics Using GaAs Nanowires and Conjugated Polymers," Nano Letters, vol. 11, pp. 408-413, 2011.

[8] C. H. Lin, K. W. Sun, Q. M. Liu, H. Shirai, C. P. Lee, "Poly (3,4ethylenedioxythiophene):poly(styrenesulfonate)/GaAs hybrid solar cells with $13 \%$ power conversion efficiency using front- and back-surface field," Opt. Express, vol. 23, pp. A1051-A1059, 2015.

[9] A. O. Patil, A. J. Heeger, F. Wudl, "Optical properties of conducting polymers," Chemical Reviews, vol. 88, pp. 183-200, 1988.

[10] J. E. Frommer, "Conducting polymer solutions," Accounts of Chemical Research, vol. 19, pp. 2-9, 1986.

[11] A. G. MacDiarmid, "Synthetic Metals," A Novel Role for Organic Polymers (Nobel Lecture), Angewandte Chemie International Edition, vol. 40, pp. 2581-2590, 2001.

[12] A. J. Heeger, "Semiconducting and Metallic Polymers: The Fourth Generation of Polymeric Materials," The Journal of Physical Chemistry B, vol. 105, pp. 8475-8491, 2001.

[13] L. Groenendaal, F. Jonas, D. Freitag, H. Pielartzik, J. R. Reynolds, "Poly(3,4-ethylenedioxythiophene) and Its Derivatives: Past, Present, and Future, Advanced Materials," vol. 12, pp. 481-494, 2000.

[14] A. J. Epstein, J. M. Ginder, F. Zuo, H. S. Woo, D. B. Tanner, A. F. Richter, M. Angelopoulos, W. S. Huang, A. G. "MacDiarmid, Insulator-to-metal transition in polyaniline: Effect of protonation in emeraldine," Synthetic Metals, vol. 21, pp. 63-70, 1987.

[15] D. Li, J. Huang, R. B. Kaner, "Polyaniline Nanofibers: A Unique Polymer Nanostructure for Versatile Applications," Accounts of Chemical Research, vol. 42, pp. 135-145, 2009.

[16] M. G. Roe, J. M. Ginder, P. E. Wigen, A. J. Epstein, M. Angelopoulos, A. G. MacDiarmid, "Photoexcitation of Polarons and Molecular Excitons in Emeraldine Base," Physical Review Letters, vol. 60, pp. 2789-2792, 1988.

[17] Y. Li, J. Hagen, D. Haarer, "Novel photoelectrochromic cells containing a polyaniline layer and a dye-sensitized nanocrystalline TiO2 photovoltaic cell," Synthetic Metals, vol. 94, pp. 273-277, 1998.

[18] Z. Hu, J. Zhang, Z. Hao, Y. Zhao, "Influence of doped PEDOT:PSS on the performance of polymer solar cells," Solar Energy Materials and Solar Cells, vol. 95, pp. 2763-2767, 2011. 
[19] T. Mustonen, K. Kordás, S. Saukko, G. Tóth, J. S. Penttilä, P. Helistö, H. Seppä, H. Jantunen, "Inkjet printing of transparent and conductive patterns of single-walled carbon nanotubes and PEDOT-PSS composites," physica status solidi (b), vol. 244, 4336-4340, 2007.

[20] M. C. Hermant, B. Klumperman, A. V. Kyrylyuk, P. van der Schoot, C. E. Koning, "Lowering the percolation threshold of single-walled carbon nanotubes using polystyrene/poly(3,4-ethylenedioxythiophene): poly(styrene sulfonate) blends," Soft Matter, vol. 5, pp. 878-885, 2009.

[21] M.-C. Hermant, P. van der Schoot, B. Klumperman, C. E. Koning, "Probing the Cooperative Nature of the Conductive Components in Polystyrene/Poly(3,4-ethylenedioxythiophene):Poly(styrene sulfonate)-Single-Walled Carbon Nanotube Composites," ACS Nano, vol. 4, pp. 2242-2248, 2010.

[22] D.-J. Yun, K. Hong, S.h. Kim, W.-M. Yun, J.-Y. Jang, W.-S. Kwon, C.-E. Park, S.-W. Rhee, "Multiwall Carbon Nanotube and Poly(3,4-ethylenedioxythiophene): Polystyrene Sulfonate (PEDOT:PSS) Composite Films for Transistor and Inverter Devices," ACS Applied Materials \& Interfaces, vol. 3, pp. 43-49, 2011.

[23] D.-J. Yun, S.-W. Rhee, "Composite Films of Oxidized Multiwall Carbon Nanotube and Poly(3,4ethylenedioxythiophene): Polystyrene Sulfonate (PEDOT:PSS) As a Contact Electrode for Transistor and Inverter Devices," ACS Applied Materials \& Interfaces, vol. 4, pp. 982-989, 2012.

[24] K. S. Novoselov, A. K. Geim, S. V. Morozov, D. Jiang, Y. Zhang, S. V. Dubonos, I. V. Grigorieva, A. A. Firsov, "Electric Field Effect in Atomically Thin Carbon Films," Science, vol. 306, pp. 666, 2004.

[25] Z. Liu, Q. Liu, Y. Huang, Y. Ma, S. Yin, X. Zhang, W. Sun, Y. Chen, Organic Photovoltaic Devices Based on a Novel Acceptor Material: Graphene, Advanced Materials, 20 (2008) 3924-3930.

[26] Y. Xu, Y. Wang, J. Liang, Y. Huang, Y. Ma, X. Wan, Y. Chen, "A hybrid material of graphene and poly (3,4ethyldioxythiophene) with high conductivity, flexibility, and transparency," Nano Research, vol. 2, pp. 343-348, 2009.

[27] G. Wang, W. Xing, S. Zhuo, "The production of polyaniline/graphene hybrids for use as a counter electrode in dyesensitized solar cells," Electrochimica Acta, vol. 66, pp. 151-157, 2012.

[28] M. Uda, A. Nakamura, T. Yamamoto, Y. Fujimoto, "Work function of polycrystalline Ag, Au and Al," Journal of Electron Spectroscopy and Related Phenomena, vol. 88-91, pp. 643-6481998.

[29] Z. R. Li, Organic Light-Emitting Materials and Devices, 2nd ed.

[30] A. Salehi, A. Nikfarjam, D. J. Kalantari, "Highly Sensitive Humidity Sensor Using Pd/Porous GaAs Schottky Contact," IEEE Sensors Journal, vol. 6, pp. 1415-1421, 2006.

[31] S. Sapp, S. Luebben, Y.B. Losovyj, P. Jeppson, D. L. Schulz, A. N. Caruso, "Work function and implications of doped poly(3,4-ethylenedioxythiophene)-co-poly(ethylene glycol)," Applied Physics Letters, vol. 88, p. 1521072006.

[32] K. A. Nagamatsu, S. Avasthi, J. Jhaveri, J. C. Sturm, “A 12\&\#x0025; Efficient Silicon/PEDOT:PSS Heterojunction Solar Cell Fabricated at \&lt; 100 \&\#x00B0;C,” IEEE Journal of Photovoltaics, vol. 4, pp. 260-264, 2014.

[33] Y. Xiao, G. Han, Y. Chang, H. Zhou, M. Li, Y. Li, “An all-solid-state perovskite-sensitized solar cell based on the dual function polyaniline as the sensitizer and p-type hole-transporting material," Journal of Power Sources, vol. 267, pp. 1-8, 2014.

[34] P. Chal, A. Shit, A. K. Nandi, "Dye-sensitized solar cell from a new organic n-type semiconductor/polyaniline composite: insight from impedance spectroscopy," Journal of Materials Chemistry C, vol. 4, pp. 272-285, 2016.

[35] J. E. Yoo, J. L. Cross, T. L. Bucholz, K. S. Lee, M. P. Espe, Y.-L. Loo, "Improving the electrical conductivity of polymer acid-doped polyaniline by controlling the template molecular weight," Journal of Materials Chemistry, vol. 17, pp. 1268-1275, 2007. 